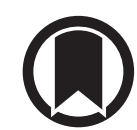

CrossMark

\section{A systematic review of clinical trial registration in major respiratory journals 2010-2018}

\author{
To the Editor:
}

The results of randomised clinical trials are regarded as the highest level of evidence for informing clinical guidelines and clinical practice $[1,2]$. The robustness of conduct and reporting of the results of randomised clinical trials is therefore of primary importance. To improve the transparency and quality of reporting of clinical trials, the International Committee of Medical Journal Editors (ICMJE) endorsed a policy on mandatory registration of clinical trials, which came into effect in July 2005 [3]. The trial registration recommendations outlined by the ICMJE state that a trial must be registered in a publicly accessible registry, before the enrolment of the first study participants, in order to be considered for publication. This policy is designed to reduce publication bias, prevent selective reporting of desirable or non-reporting of undesirable results, and reduce research waste by making the research community aware of what questions are already being addressed by active trials.

Previous studies have suggested that although ICMJE guidance recommends that journals should not publish manuscripts from trials that were not registered or were retrospectively registered, such trials continue to be published in the medical literature, including in high-impact journals [4]. While compliance with ICMJE registration has been investigated in individual specialities such as cardiology $[5,6]$, no studies to date have reported on adherence to the ICMJE policy within respiratory medicine.

This review was conducted in order to evaluate the registration practices of clinical trials published in high-impact respiratory medical journals from 2010 to 2018. Our aim was to determine the frequency of publication of unregistered and retrospectively registered trials, and to determine temporal trends in publication practices.

We conducted a systematic review based on the recommendations set in the Preferred Reporting Items for Systematic Reviews and Meta-Analyses statement [7]. This review was prospectively registered at PROSPERO (CRD42018102819).

All searches were conducted using PubMed, as all publications included are PubMed indexed. We used the sensitivity- and precision-maximising version of The Cochrane search strategy [8]. Publication date was set between January 2010 and July 2018. 2010 was chosen since mandatory trial registration was introduced in 2005 and therefore, we considered that by 2010, the majority of published trials would be eligible for registration. We studied published articles within eight journals: The Lancet Respiratory Medicine, The American Journal of Respiratory and Critical Care Medicine, Thorax, the European Respiratory Journal, Chest, Respiratory Research, Respirology and The Annals of the American Thoracic Society. These journals were chosen on the basis of impact factor, publication of original research including clinical trials and publication across all subspecialities within respiratory medicine.

No language restrictions were applied. All articles identified by search were reviewed by title and abstract, and the full text of the publication was reviewed if potentially relevant. We used the ICMJE definition of a clinical trial to determine study inclusion [9]. We excluded studies that reported on post hoc analyses or secondary analyses based on trial datasets. This study was conducted by four reviewers, two of whom were involved in both data extraction and analysis, and two were involved in the data extraction step only. Discrepancies were resolved by discussion.

@ERSpublications

Retrospective trial registration remains common in respiratory clinical trials, and may be associated with reporting bias http://ow.ly/S7p230m5kqV

Cite this article as: Suntila NSM, Smith A, Al-Dabbagh Y, et al. A systematic review of clinical trial registration in major respiratory journals 2010-2018. Eur Respir J 2018; 52: 1801491 [https://doi.org/ 10.1183/13993003.01491-2018]. 
The following data were extracted for each study: publication title, whether or not the trial was a multicentre study, region, registration number, date of enrolment of first patient, date of registration, funding source, whether the trial was academic or commercial based on the funding source, year of publication, and outcome of the trial.

Classification of trials followed a published approach used by the BMJ [4]. Briefly, a trial was considered to be registered if the publication included a registration number of an acceptable primary registry: one belonging to the World Health Organization (WHO) International Clinical Trials Registry Platform (ICTRP). If no registration number was reported in the publication, the trial was searched for using the ICTRP search platform. If no registration number was found or the trial was registered in a WHO non-compliant registry, the trial was considered "not registered".

The study start date, which the ICMJE defines as the date of enrolment of first patient [9], was recorded as that reported in the publication. The outcome of each trial was recorded as either "positive" or "negative" depending on whether or not the primary outcome measure of the trial reached statistical significance.

Trials included for further analysis were defined by their registration status as "prospective" (registration before or on the day of enrolment of first patient), "retrospective" (registration after the day of first patient enrolment) or "not registered". Retrospectively registered trials were further divided into subclasses according to the time delay (in months) between the date of enrolment of the first participant and the date of registration.

We calculated simple proportions to describe the frequency of registered and unregistered trials. Logistic regression was used to identify factors associated with trial registration status. $\mathrm{p}<0.05$ was considered statistically significant.

The search strategy identified a total of 2109 records, of which 1108 were excluded by abstract as they did not fulfil the criteria for a clinical trial. Of the 1082 potentially eligible trials, a further 158 were excluded as they did not meet the full inclusion criteria; these were mainly publications that did not report the primary results of a trial. A total of 925 studies were included for further analysis. Some publications presented the primary results of two or more trials (most of these were combinations of two identical trials due to regulatory requirements) and accounting for these, the number of trials included was 955 . These studies of multiple trials were included as single entries for our analyses.

Of the 925 studies included for our analyses, $57.1 \%$ were multicentre trials. The majority $(64.2 \%)$ of trials reported a positive outcome.

Overall, $47.7 \%$ of trials were prospectively registered and $42.2 \%$ were regarded as retrospectively registered. A total of $10.2 \%$ of trials were not registered. Excluding trials that started before mandatory registration was introduced in 2005, 51.5\% were prospectively registered, 39.0\% were retrospectively registered and 9.5\% were not registered. Examining publications by journal, The Lancet Respiratory Medicine had the highest percentage of prospectively registered trials (68.5\%) and Respirology the lowest (32.4\%) across the 8 -year study period (figure $1 \mathrm{~b}$ ). Commercially funded studies were more likely to be prospectively registered than academically funded studies ( $p=0.003$ ) (figure 1d). The proportion of retrospectively registered and unregistered studies decreased progressively from 2010 to 2018 from a mean of $72.4 \%$ in 2010 to $35.6 \%$ of trials in 2018 (figure 1c). Unregistered studies accounted for $24.6 \%$ of published studies in 2010 but were virtually eliminated by 2018 ( $0.4 \%$ of published studies) (figure 1e). In figure 1f, we show the publication factors associated with non-prospective trial publication. There was a significant effect of region $(\mathrm{p}=0.004)$ and date of publication $(\mathrm{p}=0.008)$, with failure to prospectively register trials being most common in Spain (OR 6.5, 95\% CI 2.65-15.8), followed by South America, Italy and China. Australia and New Zealand had the lowest odds of retrospective registration (OR 0.52, 95\% CI 0.27-1.01). Single-centre studies were more likely to be retrospectively registered or not registered (OR 2.14, 95\% CI 1.48-3.10). Trials in cystic fibrosis (71.1\%), pulmonary vascular disease (70.4\%), interstitial lung disease (61.5\%) and asthma (59.1\%) were most likely to be prospectively registered. Studies in sleep medicine $(35.7 \%$ and cancer $(47.6 \%)$ were least likely to be registered prospectively.

Retrospective trial registration was frequently just within 1 month of study start ( $29 \%$ of trials) but a total of $38.6 \%$ of all trials were registered $>12$ months after the first participant was enrolled (figure $1 \mathrm{~g}$ ). These trials at higher risk of bias, which are registered more than 1 year after study start date, also decreased rapidly in numbers over time (figure $1 \mathrm{~h}$ ).

To evaluate the potential for registration status to bias the literature, we examined whether registration status impacted the likelihood of a positive result. Compared to prospectively registered studies, unregistered studies were $80 \%$ more likely to have a positive result (OR 1.80, 95\% CI 1.10-2.95; $\mathrm{p}=0.02$ ). Retrospectively registered studies were $33 \%$ more likely to be positive (OR 1.33, 95\% CI 1.01-1.77; $\mathrm{p}=0.04$ ). 
a)
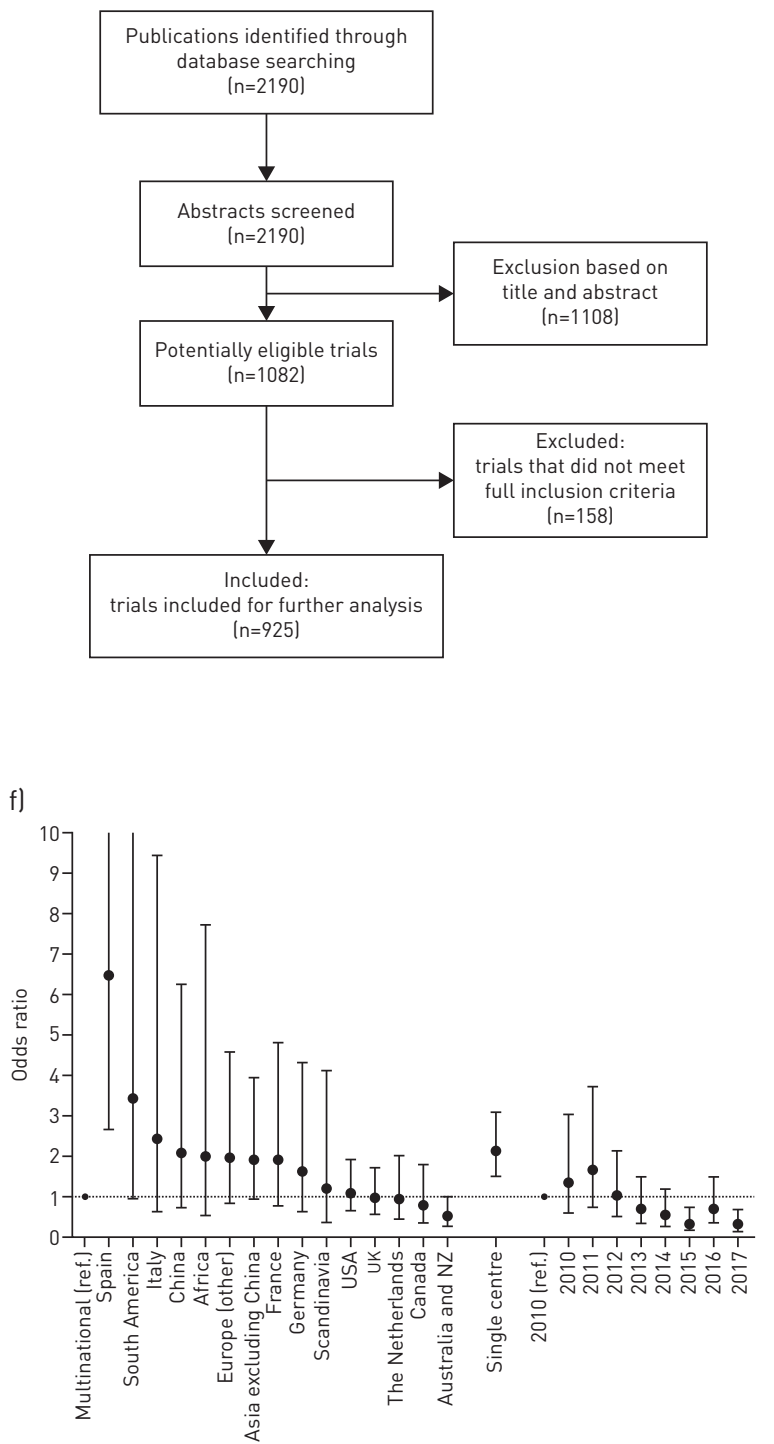

b)
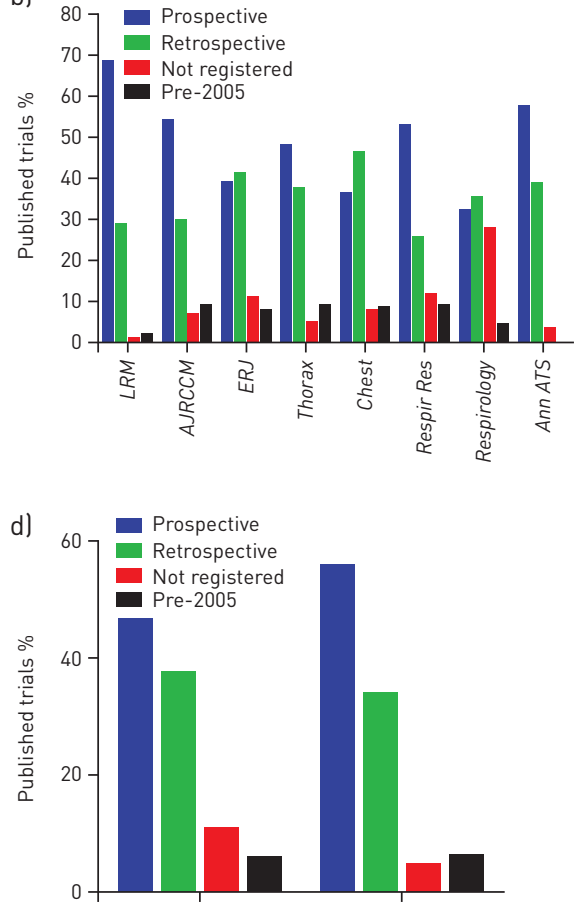

Academic

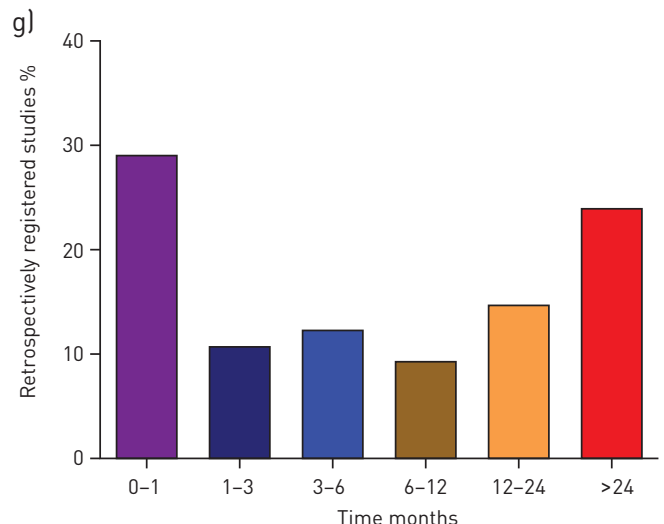

c)
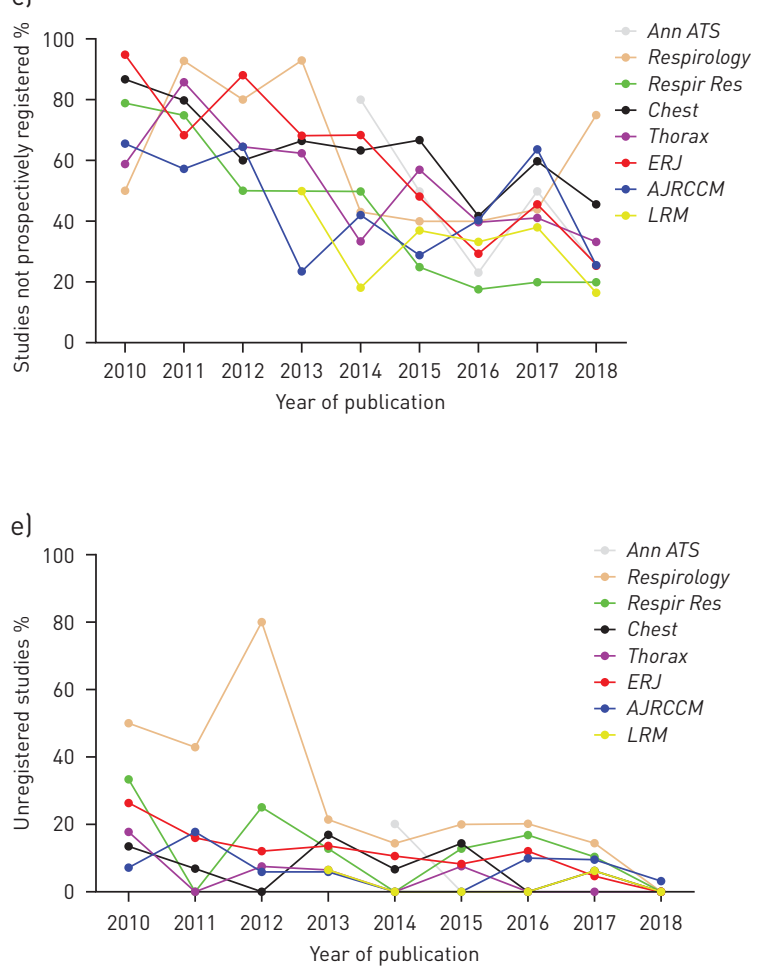

h)

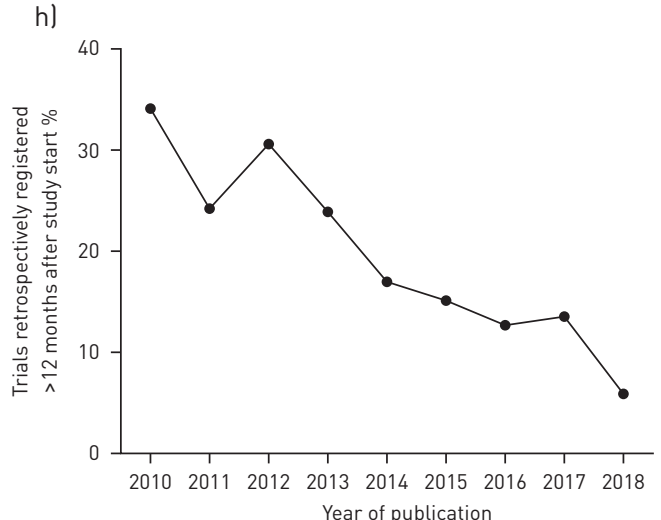

FIGURE 1 Systematic review of clinical trial registration in respiratory medicine journals 2010-2018. a) Flow chart for identification of clinical trials. b) Clinical trial registration status by journal 2010-2018. Data are presented as percentages of published trials. c) Retrospective and unregistered studies by journal over time. d) Registration status stratified by funding status. e) Percentage of unregistered trials over time. f) Forest plot showing the results of the logistic regression analysis for risk of retrospective or non-registered trials. Data are presented as odds ratios with $95 \%$ confidence intervals. The upper 95\% confidence intervals have been limited to 10 to allow easier visualisation of the overall data 9 l Timing of retrospective trial registration. h) Proportion of trials registered $>12$ months after study start date over time in all eight journals. LRM: The Lancet Respiratory Medicine: AJRCCM: The American Journal of Respiratory and Critical Care Medicine; ERJ: European Respiratory Journal; Respir Res: Respiratory Research; Ann ATS: The Annals of the American Thoracic Society; NZ: New Zealand. 
In this review of clinical trials published between 2010 and the present in eight high-impact respiratory medical journals, we found that a significant proportion of clinical trials remains retrospectively registered, years after the ICMJE introduced their policy on mandatory prospective trial registration. Additionally, we have shown that there is still a substantial number of trials that are not registered. Our results are in line with previous reports on trial registration practices in the post-implementation period of the ICMJE policy [10]. We observed a trend towards better registration compliance from 2010 to 2018, which was expected and resonates with previous findings on the improvements seen over the years in clinical trial registration [11].

There is evidence that most retrospective registration may be inadvertent [12]. Some investigators may not be aware that their trial meets the criteria for a trial that should be registered. Some investigators may be completely unaware of registration policies, although 13 years post-implementation of the ICMJE policy, this is increasingly difficult to justify [12]. Nevertheless, one of the main purposes of mandatory prospective registration is to prevent selective reporting of trial outcomes. Our finding that retrospectively registered trials are more likely to be positive has two possible explanations: either researchers may register their trial retrospectively in order to engage in selective reporting of their results or editors are more likely to ignore the requirement for prospective registration in the face of a positive trial [13]. It is therefore important that investigators, journal editors and members of research or funding organisations are all involved in addressing this issue. Journals have a responsibility to adequately check registration status at the time of submission [13]. There is evidence for the majority of retrospectively registered studies being registered before submission to a journal [10] and thus these inappropriately registered trials may go on to be published if there are no strict quality checks on the trials' registration status.

Commercially funded trials were more often registered prospectively, which may reflect more strict regulation imposed on studies with commercial interest. Studies from certain regions, including Spain, South America, Italy and China, and single-centre studies in general, were more likely to be registered inappropriately. This may be explained by variation in regional registration policies and the availability of resources for project management [11].

The quality of registration reporting has been reported in detail elsewhere $[14,15]$ but we also found inconsistencies and lack of clarity in trial reporting including failure to provide study dates in publications. We may therefore underestimate the scale of retrospective registration since we were conservative in our assessment of registration status.

In conclusion, adherence to clinical trial registration policies in respiratory medicine is improving rapidly. Publication of unregistered trials has been virtually eliminated and the challenge now is to encourage compliance with prospective registration among investigators and editors.

Nelli S.M. Suntila ${ }^{1}$, Alexandria Smith ${ }^{2}$, Yusuf Al-Dabbagh ${ }^{1}$ and James D. Chalmers ${ }^{1}$

${ }^{1}$ Scottish Centre for Respiratory Research, University of Dundee, Dundee, UK. ${ }^{2}$ University of Cambridge, Cambridge, UK.

Correspondence: James D. Chalmers, Division of Molecular and Clinical Medicine, University of Dundee, Dundee DD1 9SY, UK. E-mail: jchalmers@dundee.ac.uk

Received: Aug 062018 | Accepted after revision: Sept 122018

Conflict of interest: N.S.M. Suntila has nothing to disclose. A. Smith has nothing to disclose. Y. Al-Dabbagh has nothing to disclose. J.D. Chalmers is the deputy chief editor of the European Respiratory Journal. He has received research grants for COPD studies and personal fees from GlaxoSmithKline, Boehringer Ingelheim and Pfizer, research grants for COPD studies from AstraZeneca, research grants for bronchiectasis and personal fees from Bayer Healthcare and Grifols, consulting fees from Napp and Aradigm Corporation, and grants and personal fees from Insmed, outside the submitted work.

Support statement: J.D. Chalmers is supported by the GlaxoSmithKline/British Lung Foundation Chair of Respiratory Research.

\section{References}

1 Polverino E, Goeminne PC, McDonnell MJ, et al. European Respiratory Society guidelines for the management of adult bronchiectasis. Eur Respir J 2017; 50: 1700629.

2 Wedzicha JA, Miravitlles M, Hurst JR, et al. Management of COPD exacerbations: a European Respiratory Society/American Thoracic Society guideline. Eur Respir J 2017; 49: 1600791.

3 De Angelis C, Drazen JM, Frizelle FA, et al. Clinical trial registration: a statement from the International Committee of Medical Journal Editors. N Engl J Med 2004; 351: 1250-1251.

4 Loder E, Loder S, Cook S. Characteristics and publication fate of unregistered and retrospectively registered clinical trials submitted to The BMJ over 4 years. BMJ Open 2018; 8: e020037.

5 Scott A, Rucklidge JJ, Mulder RT. Is mandatory prospective trial registration working to prevent publication of unregistered trials and selective outcome reporting? An observational study of five psychiatry journals that mandate prospective clinical trial registration. PLoS One 2015; 10: e0133718. 
6 Emdin C, Odutayo A, Hsiao A, et al. Association of cardiovascular trial registration with positive study findings. JAMA Intern Med 2015; 175: 304.

7 Moher D, Liberati A, Tetzlaff J, et al. Preferred reporting items for systematic reviews and meta-analyses: the PRISMA statement. BMJ 2009; 339: b2535.

8 The Cochrane Collaboration. Cochrane Work. Resources. Search strategies. The Cochrane highly sensitive search strategies for identifying randomized trials in PubMed. https://work.cochrane.org/pubmed

9 International Committee of Medical Journal Editors. Recommendations. Clinical trials. 1. Registration. www.icmje. org/recommendations/browse/publishing-and-editorial-issues/clinical-trial-registration.html

10 Harriman SL, Patel J. When are clinical trials registered? An analysis of prospective versus retrospective registration. Trials 2016; 17: 187.

11 Viergever RF, Li K. Trends in global clinical trial registration: an analysis of numbers of registered clinical trials in different parts of the world from 2004 to 2013. BMJ Open 2015; 5: e008932.

12 Hunter KE, Seidler AL, Askie LM. Prospective registration trends, reasons for retrospective registration and mechanisms to increase prospective registration compliance: descriptive analysis and survey. BMJ Open 2018; 8: e019983.

13 Sotgiu G, Humbert M, Dinh-Xuan AT, et al. Clinical trials: registration and transparency. Eur Respir J 2016; 47: $1342-1344$.

14 Viergever RF, Karam G, Reis A, et al. The quality of registration of clinical trials: still a problem. PLoS One 2014; 9: e84727.

15 Huser V, Cimino JJ. Evaluating adherence to the International Committee of Medical Journal Editors' policy of mandatory, timely clinical trial registration. J Am Med Informatics Assoc 2013; 20: e169-e174.

Copyright @ERS 2018 